\title{
Inequalities for Ising Models and Field Theories which Obey the Lee-Yang Theorem*
}

\author{
Charles M. Newman \\ Department of Mathematics, Indiana University, Bloomington, Indiana, USA
}

Received September 4, 1974; in revised form October 9, 1974

\begin{abstract}
A series of inequalities for partition, correlation, and Ursell functions are derived as consequences of the Lee-Yang Theorem. In particular, the $n$-point Schwinger functions of even $\phi^{4}$ models are bounded in terms of the 2-point function as strongly as is the case for Gaussian fields; this strengthens recent results of Glimm and Jaffe and shows that renormalizability of the 2-point function by fourth degree counter-terms implies existence of a $\phi^{4}$ field theory with a moment generating function which is entire of exponential order at most two. It is also noted that if any (even) truncated Schwinger function vanishes identically, the resulting field theory is a generalized free field.
\end{abstract}

\section{The Lee-Yang Theorem}

We consider a collection of (spin) random variables $\left\{X_{j}: j=1, \ldots, N\right\}$ whose moment generating function is of the form,

$$
\boldsymbol{E}\left(\exp \left(\sum_{j} z_{j} X_{j}\right)\right)=\frac{\int \exp \left(\sum_{j} z_{j} x_{j}+\sum_{j, k} J_{j k} x_{j} x_{k}\right) \prod_{j} d \varrho_{j}\left(x_{j}\right)}{\int \exp \left(\Sigma J_{j k} x_{j} x_{k}\right) \prod_{j} d \varrho_{j}\left(x_{j}\right)},
$$

with $J_{j k} \geqq 0$ and each $\varrho_{j}$ an even probability measure such that $\int \exp \left(b x^{2}\right) d \varrho_{j}(x)<\infty$ for all real $b$. (1.1) is the (normalized) partition function for a general Ising model (with two-body ferromagnetic interactions) such as arises in the lattice approximation to even $P(\phi)$ Euclidean field models [1]. Spin- $-\frac{1}{2}$ Ising models correspond to letting each $\varrho_{j}(x)=\left(\delta\left(x_{j}-1\right)+\delta\left(x_{j}+1\right)\right) / 2$.

A very general version of the Lee-Yang theorem which applies to spin- $\frac{n}{2}$ as well as to $d \varrho_{j} / d x=C_{j} \exp \left(-a_{j} x^{4}-b_{j} x^{2}\right)$ (corresponding to an even $\phi^{4}$ field theory) is as follows $[2,3,4]$ :

Theorem 1. If for each $j$, the zeros of $\int \exp (z x) d \varrho_{j}(x)$ are pure imaginary, then for any choice of $\lambda_{j} \geqq 0$, the zeros of $\boldsymbol{E}\left(\exp \left(z \Sigma \lambda_{j} X_{j}\right)\right)$ are pure imaginary.

The Lee-Yang theorem has been widely used in both statistical mechanics and quantum field theory [e.g., 5,6] for the investigation of phase transition phenomena; it will be applied in this paper to obtain correlation inequalities and related results which in the case of field theory should be useful in proving existence and studying triviality (or non-triviality) of $\phi^{4}$ models. We note that perturbation theory predicts the applicability of our results (i.e., Theorem 10 below) in spacetime dimension less than 5 .

* Supported in part by the Indiana University Foundation and by the National Science Foundation under Grant NSF-GP-24003. 
We will call $\left\{X_{j}\right\}$, as defined by (1.1), an Ising model of Lee-Yang type if each $\varrho_{j}$ satisfies the hypotheses of Theorem $1 .\left\{X_{j}\right\}$ will be said to be of $\phi^{4}$ type if for each $j, d \varrho_{j} / d x=C_{j} \exp \left(-a_{j} x^{4}-b_{j} x^{2}\right)$ with $a_{j}>0$. The remainder of this paper concerns only models of Lee-Yang type: Section 2 deals with individual random variables, Section 3 with Ising models and Section 4 with random fields (Euclidean quantum fields).

\section{Random Variables of Type $\mathscr{L}$}

We define a random variable $X$ to be of type $\mathscr{L}$, if for some $C$ and $C^{\prime}, \mid E(\exp$ $(z X)) \mid \leqq C \exp \left(C^{\prime}|z|^{2}\right)$ for all $z$, and $\boldsymbol{E}(\exp (z X))$ is even with only pure imaginary zeros. Theorem 1 shows that for an Ising model of Lee-Yang type, $X(\vec{\lambda}) \equiv \Sigma \lambda_{j} X_{j}$ is of type $\mathscr{L}$ if all $\lambda_{j} \geqq 0$ (or by symmetry, if all $\lambda_{j} \leqq 0$ ).

The moments, $s_{n}$, and cumulants, $u_{n}$, of a random variable $X$ may be defined in the usual way by,

$$
\boldsymbol{E}(\exp (z X))=\sum_{n=0}^{\infty} \frac{s_{n}}{n !} z^{n}=\exp \left(\sum_{n=1}^{\infty} \frac{u_{n}}{n !} z^{n}\right) .
$$

The following proposition is basic to all other results.

Proposition 2. If $X$ is of type $\mathscr{L}$, then

$$
\boldsymbol{E}(\exp (z X))=\exp \left(b z^{2}\right) \prod_{j}\left(1+\left(z / \alpha_{j}\right)^{2}\right)
$$

for some $b \geqq 0$ and $0<\alpha_{1} \leqq \alpha_{2} \ldots$, with $\Sigma\left(1 / \alpha_{j}\right)^{2}<\infty$; here, $\left\{\alpha_{j}\right\}$ may be empty, finite, or infinite.

Proof. Except for the statement that $b \geqq 0$, this follows immediately from the Hadamard factorization theorem [7, Theorems 2.7.1 and 2.10.1] as in [3]. To see that $b \geqq 0$, we note that (2.2) implies that for any $\varepsilon>0$, and real $r, \boldsymbol{E}(\exp (\mathrm{rX}))$ $\leqq \exp \left((b+\varepsilon) r^{2}\right)$ for sufficiently large $|\mathrm{r}|$; thus if $b<0, \boldsymbol{E}(\exp (r X)) \rightarrow 0$ as $r \rightarrow \pm \infty$ which is impossible for any random variable $X$.

Q.E.D.

The following theorems are extraordinarily simple consequences of Proposition 2; we first extend a result concerning the cumulants (Ursell functions) which was originally stated in $[3,4]$.

Theorem 3. If $X$ is of type $\mathscr{L}$, then $u_{2 m-1}=0$ and $(-1)^{m-1} u_{2 m} \geqq 0$ for $m=1,2, \ldots$. If $u_{2 m}=0$ for any $m=1,2, \ldots$, then $u_{n}=0$ for all $n>2$ and $X$ is Gaussian.

Proof. We combine (2.1), (2.2), and the expansion $\log \left(1+w^{2}\right)=w^{2}-\frac{1}{2} w^{4}$ $+\frac{1}{3} w^{6}-\ldots$ for $w=\left(z / \alpha_{j}\right)$ to derive that $u_{2 m-1}=0$,

$$
s_{2}=u_{2}=2\left(b+\sum_{j}\left(1 / \alpha_{j}\right)^{2}\right)
$$

and for $m \geqq 2$,

$$
u_{2 m}=(-1)^{m-1} \frac{(2 m) !}{m} \sum_{j}\left(1 / \alpha_{j}\right)^{2 m}
$$

Thus $(-1)^{m-1} u_{2 m} \geqq 0 ;$ and $u_{2 m}=0 \Rightarrow\left\{\alpha_{j}\right\}$ is empty so that $\boldsymbol{E}(\exp (z X))=\exp \left(b z^{2}\right)$. 
Remark 1. The second part of Theorem 3 may be thought of as a strong version (for the class $\mathscr{L}$ ) of the Marcinkiewicz Theorem [e.g.: 8, p. 58] which (loosely) states that, if for some $n_{0}, u_{n}=0$ for all $n \geqq n_{0}$, then $X$ is Gaussian.

Theorem 4. If $X$ is of type $\mathscr{L}$, then for any $k=0,1,2, \ldots$, and real $r$,

$$
\exp \left(\sum_{n=1}^{4 k} \frac{u_{n}}{n !} r^{n}\right) \leqq \boldsymbol{E}(\exp r X) \leqq \exp \left(\sum_{n=1}^{4 k+2} \frac{u_{n}}{n !} r^{n}\right)
$$

thus, for complex $z$,

$$
|\boldsymbol{E}(\exp z X)| \leqq \exp \left(\frac{1}{2} S_{2} \cdot(\operatorname{Re} z)^{2}\right) .
$$

Proof. This theorem follows immediately from (2.1), (2.2), (2.3), (2.4), and the simple fact that for $a \geqq 0$,

$$
\sum_{m=1}^{2 k} \frac{(-1)^{m-1}}{m} a^{m} \leqq \log (1+a) \leqq \sum_{m=1}^{2 k+1} \frac{(-1)^{m-1}}{m} a^{m}
$$

for any $k=0,1,2, \ldots$ Inequality (2.7) is most easily seen by noting that

$$
\log (1+a)=\int_{0}^{a} \frac{1}{1+x} d x
$$

while

$$
\sum_{m=1}^{j} \frac{(-1)^{m-1}}{m} a^{m}=\int_{0}^{a} \frac{1+(-1)^{j+1} x^{j}}{1+x} d x .
$$

Theorem 5. If $X$ is of type $\mathscr{L}$, then for $m=1,2, \ldots, s_{2 m-1}=0=u_{2 m-1}$, while

$$
0 \leqq s_{2 m} \leqq \frac{(2 m) !}{2^{m} m !}\left(s_{2}\right)^{m}, 1
$$

and

$$
0 \leqq(-1)^{m-1} u_{2 m} \leqq \frac{(2 m) !}{2^{m} m}\left(s_{2}\right)^{m}
$$

Proof. We first note that (2.9) follows easily from (2.3), (2.4), and the fact that

$$
\Sigma\left(1 / \alpha_{j}\right)^{2 m} \leqq\left(\Sigma\left(1 / \alpha_{j}\right)^{2}\right)^{m} \leqq\left(s_{2} / 2\right)^{m} .
$$

To prove (2.8), we note that since each Taylor coefficient of $\left(1+\left(r / \alpha_{j}\right)^{2}\right)$ is bounded above by the corresponding Taylor coefficient of $\exp \left(\left(r / \alpha_{j}\right)^{2}\right)$, it follows from (2.1) and (2.2), that each moment of $X$ is bounded above by the corresponding moment of a (Gaussian) random variable with moment generating function

$$
\exp \left(\left(b+\Sigma\left(1 / \alpha_{j}\right)^{2}\right) z^{2}\right)
$$

the $2 m^{\text {th }}$ moment of this Gaussian variable is of course just $\frac{(2 m) !}{m !}\left(b+\Sigma\left(1 / \alpha_{j}\right)^{2}\right)^{m}$ which together with (2.3) yields the desired result.

Q.E.D.

${ }^{1}$ In the special case when $X$ is a linear combination of independent Bernoulli (spin- $\frac{1}{2}$ ) random variables, this bound is known as Khintchine's inequality (see chap. 5 of [20]). 
Remark 2. For Gaussian $X$, both the upper bound of (2.8) (for all $m$ ) and the lower bound of (2.9) (for $m \geqq 2$ ) are equalities. On the other hand if $X$ is not identically zero then neither the lower bound of (2.8) (for any $m$ ) nor the upper bound of (2.9) (for any $m \geqq 2$ ) can be an equality; this is trivial for (2.8) and follows for (2.9) from the fact that equality of the upper bound can only happen when $\boldsymbol{E}(\exp (z X))$ $=1+(z / \alpha)^{2}$ which is not the moment generating function of any random variable.

To simplify the statement of the next theorem, we define for $m=1,2, \ldots$, $v_{2 m}=(-1)^{m-1} \frac{m}{(2 m) !} u_{2 m} ;$ thus $v_{2}=b+\Sigma\left(1 / \alpha_{j}\right)^{2}$ while for $m>1, v_{2 m}=\Sigma\left(1 / \alpha_{j}\right)^{2 m}$.

Theorem 6. Suppose $X$ is of type $\mathscr{L}$; then for any even integers $n, n_{1}, \ldots, n_{k}$ with $n=\beta_{1} n_{1}+\cdots+\beta_{k} n_{k}, \beta_{j} \geqq 0$ for all $j$, and $\beta_{1}+\cdots+\beta_{k} \geqq 1$,

$$
v_{n} \leqq \prod_{j=1}^{k}\left(v_{n_{j}}\right)^{\beta_{j}} .
$$

In particular,

$$
v_{n} \leqq v_{n_{1}} \cdot v_{n_{2}} \text { for } n=n_{1}+n_{2}
$$

while for $n_{1}<n<n_{2}$, we let $\beta=\frac{n_{2}-n}{n_{2}-n_{1}}$, so that

$$
v_{n} \leqq\left(v_{n_{1}}\right)^{\beta}\left(v_{n_{2}}\right)^{1-\beta} \text {. }
$$

Proof. If $b=0$, it follows directly from Hölder's inequality that $\Pi\left(v_{n_{j}}\right)^{\beta_{j}}$ $\geqq\left(\Sigma\left(1 / \alpha_{i}\right)^{n^{\prime}}\right)^{p}$ where $p=\Sigma \beta_{j}$ and $n^{\prime}=n / p ;(2.10)$ then follows from the inequality $\left(\right.$ taking $\left.y_{i}=\left(1 / \alpha_{i}\right)^{n^{\prime}}\right)$ :

$$
\left(\Sigma\left|y_{i}\right|^{p}\right)^{1 / p} \leqq \Sigma\left|y_{i}\right|
$$

If $b \neq 0$, we define $v_{n}(M)=M(\sqrt{b / M})^{n}+\Sigma\left(1 / \alpha_{j}\right)^{n}$ and note that $v_{n}(M) \rightarrow v_{n}$ as $M \rightarrow \infty$ for $n=2,4, \ldots$. Since the above proof (for $b=0$ ) implies the desired inequalities for $v_{n}(M)$ (for integral $M$ ), the proof of the theorem is completed by letting $M \rightarrow \infty$.

Q.E.D.

Theorem 7. If $\pm i \alpha_{1}$ are the nearest zeros to the origin of $\boldsymbol{E}(\exp (z X))$ for a random variable $X$ of type $\mathscr{L}$; then for $m^{\prime}>m \geqq 1$,

$$
\alpha_{1} \leqq\left(\frac{m\left(2 m^{\prime}\right) !}{m^{\prime}(2 m) !} \frac{(-1)^{m-1} u_{2 m}}{(-1)^{m^{\prime}-1} u_{2 m^{\prime}}}\right)^{1 /\left(2 m^{\prime}-2 m\right)}
$$

In particular,

$$
\alpha_{1}^{2} \leqq \frac{6 s_{2}}{\left|u_{4}\right|} .
$$

Proof. Since $\alpha_{1} \leqq \alpha_{j}$, it follows that

$$
\Sigma\left(1 / \alpha_{j}\right)^{2 m^{\prime}} \leqq\left(1 / \alpha_{1}\right)^{\left(2 m^{\prime}-2 m\right)} \Sigma\left(1 / \alpha_{j}\right)^{2 m}
$$

which together with (2.3) and (2.4) yields (2.13).

Q.E.D. 


\section{Ising Models of Lee-Yang Type}

We now return to general Ising models, $\left\{X_{j}\right\}$, satisfying the hypotheses of Theorem 1 . The correlations, $S_{n}$, and Ursell functions, $U_{n}$, are symmetric $n$-linear functions on $C^{n}$, and may be defined by,

$$
\begin{aligned}
\boldsymbol{E}(\exp (X(\vec{z}))) & =\sum_{n=0}^{\infty} \frac{1}{n !} S_{n}(\vec{z}, \ldots, \vec{z}) \\
& =\exp \left(\sum_{n=1}^{\infty} \frac{1}{n !} U_{n}(\vec{z}, \ldots, \vec{z})\right) ;
\end{aligned}
$$

we also consider the point correlations $S_{n}\left(j_{1}, \ldots, j_{n}\right)$ defined by

$$
S_{n}(\vec{z}, \ldots, \vec{z}) \equiv \sum_{j_{1}, \ldots, j_{n}=1}^{N} S_{n}\left(j_{1}, \ldots, j_{n}\right) z_{j_{1}} \cdots z_{j_{n}}
$$

and similarly for $U_{n}$. Since $X(\vec{\lambda})$ is of type $\mathscr{L}$ for $\vec{\lambda} \geqq 0$ (or $\vec{\lambda} \leqq 0$ ), all the results of Section 2 apply immediately to Ising models of Lee-Yang type; we next extend and amplify certain of them.

Theorem 8. For any $\vec{\lambda} \geqq 0,(-1)^{m-1} U_{2 m}(\vec{\lambda}, \ldots, \vec{\lambda}) \geqq 0$. If for any $m=1,2, \ldots$, $U_{2 m}\left(j_{1}, \ldots, j_{2 m}\right) \equiv 0$, then $\left\{\bar{X}_{j}\right\}$ is a (jointly) Gaussian family of random variables.

Proof. The first statement of the theorem and the fact that $U_{2 m} \equiv 0$ implies that $X(\vec{\lambda})$ is Gaussian for $\vec{\lambda} \geqq 0$ follow immediately from Theorem 3 . To see that $\left\{X_{j}\right\}$ is jointly Gaussian, we note that when $\vec{\lambda} \geqq 0, \boldsymbol{E}(\exp (X(\vec{\lambda})))$ is of the form $\exp \left(\Sigma B_{i j} \lambda_{i} \lambda_{j}\right)$; since it is an entire function of $\vec{\lambda}$, it must be of this form for all $\vec{\lambda}$, which completes the proof.

Q.E.D.

Before stating the next theorem, we note that for a large class of general Ising models (including spin- $\frac{n}{2}$ and $\phi^{4}$ type), it has been shown that $U_{4}\left(j_{1}, \ldots, j_{4}\right) \leqq 0$ for any $j_{1}, \ldots, j_{4}[9]$ and it has been conjectured $[4,10]$ that in general $(-1)^{m-1} U_{2 m}$ $\left(j_{1}, \ldots, j_{2 m}\right) \geqq 0$. Based upon the methods and results of Theorems 3,5 , and 6 , other similar conjectures can be made for such Ising models: For any choice of $1 \leqq m_{1}<m_{2}<\cdots<m_{k}$ and $n_{1}, \ldots, n_{k} \geqq 1$, with $m=\Sigma n_{i} m_{i}, n=\Sigma n_{i}$, it can be conjectured that

$$
0 \leqq S_{2 m}(1, \ldots, 2 m) \leqq \Sigma^{\prime} S\left(\Lambda_{1}\right) \cdots S\left(\Lambda_{n}\right)
$$

and

$$
\begin{aligned}
0 \leqq & (-1)^{m-1} U_{2 m}(1, \ldots, 2 m) \\
& \leqq \frac{(-1)^{m-n} \prod_{i=1}^{k}\left(m_{i}\right)^{n_{1}}\left(n_{i}\right) !}{m} \Sigma^{\prime} U\left(\Lambda_{1}\right) \ldots U\left(\Lambda_{n}\right),
\end{aligned}
$$

where $\Sigma^{\prime}$ denotes the sum over the $(2 m) ! /\left(\prod_{i=1}^{k}\left(\left(2 m_{i}\right) !\right)^{n_{i}} n_{i} !\right)$ unordered partitions of $\{1, \ldots, 2 m\}$ into $n$ disjoint sets $\Lambda_{1}, \ldots, \Lambda_{n}$, exactly $n_{i}$ of which contain $2 m_{i}$ elements and where $S\left(\left\{j_{1}, \ldots, j_{r}\right\}\right) \equiv S_{r}\left(j_{1}, \ldots, j_{r}\right)$ (and similarly for $U$ ). Inequality (3.6) of the following theorem is a weak version of (3.3) for the case $n_{1}=m, m_{1}=1, k=1$. 
Theorem 9. For $\vec{z}, \vec{z}^{1}, \ldots, \vec{z}^{2 m} \in \mathbb{C}^{N}$,

$$
\begin{aligned}
|\boldsymbol{E}(\exp (X(\vec{z})))| & \leqq|\boldsymbol{E}(\exp (X(|\operatorname{Re} \vec{z}|)))| \\
& \leqq \exp \left(\frac{1}{2} S_{2}(|\operatorname{Re} \vec{z}|,|\operatorname{Re} \vec{z}|)\right),
\end{aligned}
$$

while

$$
\left|S_{2 m}\left(\vec{z}^{1}, \ldots, \vec{z}^{2 m}\right)\right| \leqq \frac{(2 m) !}{2^{m} m !} \prod_{j=1}^{2 m}\left(S_{2}\left(\left|\vec{z}^{j}\right|,\left|\vec{z}^{j}\right|\right)\right)^{\frac{1}{2}},
$$

where $|\vec{z}|=\left(\left|z_{1}\right|, \ldots,\left|z_{N}\right|\right)$ and $|\operatorname{Re} \vec{z}|=\left(\left|\operatorname{Re} z_{1}\right|, \ldots,\left|\operatorname{Re} z_{N}\right|\right)$.

Proof. Griffith's first inequality $[11,12]$ states that $S_{n}\left(j_{1}, \ldots, j_{n}\right) \geqq 0$ for all $j_{1}, \ldots, j_{n}$ so that clearly $\left|S_{n}\left(\vec{z}^{1}, \ldots, \vec{z}^{n}\right)\right| \leqq S_{n}\left(\left|\vec{z}^{1}\right|, \ldots,\left|\vec{z}^{n}\right|\right)$ and $\boldsymbol{E}(\exp (X(\operatorname{Re} \vec{z})))$ $\leqq E(\exp (X(|\operatorname{Re} \vec{z}|)))$. The theorem then follows from Theorems 4 and 5 together with the fact ${ }^{2}$ that for real $\vec{\lambda}^{j}$,

$$
\left|S_{2 m}\left(\vec{\lambda}^{1}, \ldots, \vec{\lambda}^{2 m}\right)\right| \leqq \prod_{j=1}^{2 m}\left(S_{2 m}\left(\vec{\lambda}^{j}, \ldots, \vec{\lambda}^{j}\right)\right)^{1 / 2 m}
$$

(3.7) may be derived by expressing

$$
\left|S_{2 m}\left(\vec{\lambda}^{1}, \ldots, \vec{\lambda}^{2 m}\right)\right|=\left|\boldsymbol{E}\left(\prod_{j=1}^{2 m} X\left(\vec{\lambda}^{j}\right)\right)\right|
$$

then using Hölder's inequality to bound the right hand side of (3.8) by

$$
\left\{\boldsymbol{E}\left(\left(X\left(\vec{\lambda}^{1}\right)\right)^{2 m}\right\}^{1 / 2 m} \cdot\left\{\boldsymbol{E}\left(\prod_{j=2}^{2 m}\left|X\left(\vec{\lambda}^{j}\right)\right|^{2 m / 2 m-1}\right)\right\}^{(2 m-1) / 2 m}\right.
$$

and continuing to use Hölder's inequality on the second factor of (3.9) another $(2 m-2)$ times.

Q.E.D.

\section{Random Fields of Lee-Yang Type}

To explain the relevance of Theorem 9 to the construction of $\phi^{4}$ field theories, we mimic the results of [13]. We define a lattice field of Lee-Yang type to be a random field $\phi(f)$ indexed by $f \in \mathscr{S}\left(\mathbb{R}^{d}\right)$, the Schwartz space of rapidly decreasing $C^{\infty}$ test functions, such that $\phi(f)=\sum_{i=1}^{N} f\left(y_{i}\right) X_{i}$ for some fixed choice of $y_{1}, \ldots, y_{N}$ $\in \mathbb{R}^{d}$ where $\left\{X_{i}\right\}$ is an Ising model of Lee-Yang type.

The Schwinger functions, $S_{n}$, and truncated Schwinger functions, $U_{n}$, of a random field are symmetric tempered distributions on $\left(\mathbb{R}^{d}\right)^{n}$ and can be defined as usual by

$$
\boldsymbol{E}(\exp (\phi(f)))=\sum_{n=0}^{\infty} \frac{1}{n !} S_{n}(f, \ldots, f)=\exp \left(\sum_{n=1}^{\infty} \frac{1}{n !} U_{n}(f, \ldots, f)\right) .
$$

Theorem 10. If $\left\{\phi_{k}\right\}$ is an infinite sequence of lattice fields of Lee-Yang type and

$$
\left|\boldsymbol{E}\left(\phi_{k}(f) \phi_{k}(g)\right)\right| \leqq\|f\|\|g\|
$$

\footnotetext{
${ }^{2}$ The suggestion to use (3.7) to derive (3.6) is due to B. Simon.
} 
for some fixed $\mathscr{S}$-norm $\|\cdot\|$, then $\|\cdot\|$ may be taken as

$$
\|f\|=C \sup _{y \in \mathbb{R}^{d}}\left|\left(1+|y|^{2}\right)^{r} f(y)\right|
$$

for some choice of $C$ and $r$. In addition, there exists a subsequence, $k_{j} \rightarrow \infty$, and a random field $\phi$ indexed by $f \in F$, the Banach space of continuous functions with finite $\|\cdot\|$-norm, such that

and

$$
\boldsymbol{E}(\exp (\phi(f)))=\lim _{j \rightarrow \infty} \boldsymbol{E}\left(\exp \left(\phi_{k_{j}}(f)\right)\right)
$$

$$
S_{n}\left(f_{1}, \ldots, f_{n}\right) \equiv \boldsymbol{E}\left(\phi\left(f_{1}\right) \ldots \phi\left(f_{n}\right)\right)=\lim _{j \rightarrow \infty} \boldsymbol{E}\left(\phi_{k_{j}}\left(f_{1}\right) \ldots \phi_{k_{j}}\left(f_{n}\right)\right)
$$

for any $f, f_{1}, \ldots, f_{n} \in F$. Further, $\boldsymbol{E}(\exp (\phi(f)))$ is an even entire functional on $F$ such that

and

$$
\begin{aligned}
|\boldsymbol{E}(\exp (\phi(f)))| & \leqq \exp \left(\frac{1}{2} S_{2}(|\operatorname{Re} f|,|\operatorname{Re} f|)\right) \\
& \leqq \exp \frac{1}{2}\|f\|^{2},
\end{aligned}
$$

$$
\begin{aligned}
\left|S_{2 m}\left(f_{1}, \ldots, f_{2 m}\right)\right| & \leqq \frac{(2 m) !}{2^{m} m !} \prod_{j=1}^{2 m}\left(S_{2}\left(\left|f_{j}\right|,\left|f_{j}\right|\right)\right)^{\frac{1}{2}} \\
& \leqq \frac{(2 m) !}{2^{m} m !} \prod_{j=1}^{2 m}\left\|f_{j}\right\| .
\end{aligned}
$$

Finally, if $f \geqq 0, \phi(f)$ is a random variable of type $\mathscr{L}$ so that Theorems 2 through 7 apply; in particular if $U_{2 m}$ is identically zero for any $m=1,2, \ldots$, then $\phi$ is a Gaussian random field.

Proof. The fact that $\|\cdot\|$ may be taken as in (4.3) is proven in [13, Prop. 2] and is based on Griffiths' first inequality which shows that $S_{2}$ (or $S_{2}^{(k)}$ ) is a positive measure on $\left(\mathbb{R}^{d}\right)^{2}$. We define analytic functionals as in [14, Appendix] and note that for each $k, \boldsymbol{E}\left(\exp \left(\phi_{k}(f)\right)\right)$ is entire on $F$ and, by (3.5) and (4.2), is bounded uniformly in $k$ on sets of bounded $F$-norm. By Proposition A3 of [14, Appendix], $\mathscr{E}(f)$, defined as the right hand side of (4.4), will exist and be entire if $k_{j}$ can be chosen so that the right hand side of (4.4) converges on a dense subset of $F$; since $F$ is separable, this can be done by a standard diagonalization procedure. It also follows from Proposition A3 of [14] that (4.5) is correct with the left hand side replaced by an appropriate derivative of $\mathscr{E}(f)$; thus (4.4), (4.5), (4.6), and (4.7) all follow once we see that $\mathscr{E}(f)=\boldsymbol{E}(\exp (\phi(f)))$ for some random field $\phi$. Now by Minlos' theorem [15, Chap. 4], there exists a probability measure $\mu$ on $\mathscr{S}^{\prime}\left(\mathbb{R}^{d}\right)$ such that $\mathscr{E}(f)=\int \exp (T(f)) d \mu(T)$ and thus for $f \in \mathscr{S}$, we may define $\phi(f)=T(f)$ on the probability space $\left(\mathscr{S}^{\prime}, \mu\right)$; it then follows from (4.6) and (4.7) that, as $f_{j} \rightarrow f$ in $F$ with $f_{j} \in \mathscr{S}, \phi\left(f_{j}\right)$ converges (in $L^{p}\left(\mathscr{S}^{\prime}, \mu\right)$ for $\left.1 \leqq p<\infty\right)$ to some random variable we call $\phi(f)$ with $\boldsymbol{E}(\exp (\phi(f)))=\mathscr{E}(f)$.

To see that $\phi(f)$ is of type $\mathscr{L}$ when $f \geqq 0$, we merely note that $\phi_{k_{j}}(f)$ is of type $\mathscr{L}$ for all $j$ and that $\boldsymbol{E}\left(\exp \left(z \phi_{k_{j}}(f)\right)\right)$ converges uniformly on compacts in $\mathbb{C}$ so that by Hurwitz' theorem, $\boldsymbol{E}(\exp (z \phi(f)))$ has only pure imaginary zeros in $z$ (and is of the desired exponential order by (4.6)). The final statement of the theorem 
concerning $U_{2 m}$ follows directly from Theorem 3, the fact that any $f \in F$ can be written as the difference of two nonnegative functions in $F$, and the proof of Theorem 8.

Q.E.D.

Remark 3. If the $\phi_{k}$ are lattice approximations to an even $\phi^{4}$ field theory [1], then it follows as in [13] that the Schwinger functions of $\phi$ satisfy the OsterwalderSchrader axioms [16] except possibly for Euclidean covariance and clustering. Specifically, we mean that with the added assumption of translation invariance, it follows from (4.7) and Griffiths' first inequality that the Schwinger functions (expressed in terms of difference variables) satisfy axiom $\boldsymbol{E} 0^{\prime}$ of $[16$, p. 81].

Remark 4. The fact that the vanishing of $U_{2 m}$ for a single $m$ implies that $\phi$ is a generalized free field should be compared with Robinson's application [17] of the Marcinkiewicz theorem (see Remark 1 above) from which he derived the same conclusion based on the assumption that all truncated vacuum expectation values vanish beyond a certain order.

For our last theorem, we consider $\phi^{4}$ models for which it is known that $U_{4}\left(y_{1}, y_{2}, y_{3}, y_{4}\right) \leqq 0[9 ; 18$, p. 172]. It then follows from Theorem 10 that $\int U_{4} d y_{1} d y_{2} d y_{3} d y_{4}=0$ implies that $U_{4} \equiv 0$ and thus that the corresponding $\phi$ is a generalized free field (i.e., Gaussian); we state this fact in more picturesque language as Theorem 11 below.

If $\phi$ is translation invariant and $\chi \equiv \int S_{2}\left(y_{1}, y_{2}\right) d y_{1}<\infty$, we define $g_{0}$ the (dimensional) physical coupling constant as

$$
g_{0}=\chi^{-4} \int U_{4}\left(y_{1}, y_{2}, y_{3}, y_{4}\right) d y_{1} d y_{2} d y_{3}
$$

(the dimensionless physical coupling constant is $g \equiv g_{0} / m^{d-4}$ where $d$ is the space-time dimension, and $m \geqq 0$ is the physical mass) [19]. We then have as a corollary to Theorem 10 the eminently believable result that the physical coupling constant only vanishes for a (generalized) free field:

Theorem 11. Suppose $\phi$ is a translation invariant random field obtained as a limit (in the sense of Theorem 10) of even lattice fields of $\phi^{4}$ type. If $\chi<\infty$ and $g_{0}=0$ (or if $\chi^{4} m^{d-4}<\infty$ and $g=0$ ), then $\phi$ is a generalized free field (i.e., Gaussian).

\section{References}

1. Guerra,F., Rosen, L., Simon, B.: The $P(\phi)_{2}$ Euclidean quantum field theory as classical statistical mechanics. Ann. Math. (to appear)

2. Simon, B., Griffiths, R.: Commun. math. Phys. 33, 145-164 (1973)

3. Newman, C. M.: Comm. Pure Appl. Math. 27, 143-159 (1974)

4. Newman, C.M.: Nondiscrete spins and the Lee-Yang theorem. In: Velo, G., Wightman, A.S. (Eds.): Constructive quantum field theory. Berlin-Heidelberg-New York: Springer 1973

5. Ruelle, D.: Statistical mechanics — rigorous results. New York: Benjamin 1969

6. Simon, B.: The $P(\phi)_{2}$ Euclidean (quantum) field theory. Princeton: Princeton University Press 1974

7. Boas, R.P.: Entire functions. New York: Academic Press 1954

8. Linnik, Yu.V.: Decomposition of probability distributions. London: Oliver and Boyd 1964

9. Lebowitz, J.: Commun. math. Phys. 35, 87-92 (1974)

10. Feldman, J.: Canadian J. Phys. 52, 1583-1587 (1974)

11. Griffiths, R. B.: J. Math. Phys. 8, 484-489 (1967)

12. Kelly, D. G., Sherman, S. : J. Math. Phys. 9, 466-484 (1968)

13. Glimm, J., Jaffe, A.: Phys. Rev. Letters 33, 440—442 (1974) 
14. Glimm, J., Jaffe,A.: The entropy principle for vertex functions in quantum field models. To appear in Ann. Inst. H. Poincaré

15. Gelfand,I.M., Vilenkin, N. Ya.: Generalized functions, Vol. 4. New York: Academic Press 1964

16. Osterwalder, K.: Euclidean Green's functions and Wightman distributions. In: Velo, G., Wightman,A.S. (Eds.): Constructive quantum field theory. Berlin-Heidelberg-New York: Springer 1973

17. Robinson, D. W.: Commun. math. Phys. 1, 89-94 (1965)

18. Glimm, J., Jaffe,A., Spencer, T.: The particle structure of the weakly coupled $P(\phi)_{2}$ model and other applications of high temperature expansions. In: Velo, G., Wightman, A.S. (Eds.): Constructive quantum field theory. Berlin-Heidelberg-New York: Springer 1973

19. Glimm,J., Jaffe,A.: Absolute bounds on vertices and couplings. Rockefeller/Harvard preprint (1974)

20. Zygmund, A.: Trigonometrical series, new ed., Vol. 1. Cambridge: Cambridge Univ. Press 1959

Communicated by A.S. Wightman

\author{
Charles M. Newman \\ Department of Mathematics \\ Indiana University \\ Bloomington, Indiana 47401, USA
}

Note Added in Proof. A minor alteration in the proof of Theorem 4 shows that the magnetization as a function of external field, $\boldsymbol{E}(X \exp r X) / \boldsymbol{E}(\exp r X)$, has an alternating bound property with respect to its Taylor expansion analogous to (2.5). We also note that extensions of certain results of this article to models in a positive external field will appear in a paper by the author in the Journal of Mathematical Physics. Finally, we mention that the author has obtained a proof of (3.3) and related inequalities by using graphical techniques of Kelly and Sherman [12]. 
